\title{
Caracterización y variabilidad química de dos aceites esenciales de Croton funckianus (Euphorbiaceae)
}

\author{
Characterization and Chemical Variability of Two Essential Oils From Croton \\ funckianus (Euphorbiaceae)
}

\author{
C. A. Coy Barrera ${ }^{a}$,* \\ D. C. Gómez ${ }^{\mathrm{a}}$
}

Recepción: 04-may-15

Aceptación: 07-jun-15

\begin{abstract}
Resumen
Para caracterizar químicamente el aceite esencial obtenido tanto de hojas como de frutos de dos muestras de la especie Croton funckianus, colectadas en dos sitios diferentes (Fusagasugá y Bogotá), se realizó el análisis cromatográfico por la técnica de cromatografía de gases acoplada a espectrometría de masas (CG-EM), y posteriormente se compararon los perfiles obtenidos, determinando algunas diferencias para el caso de las hojas y un alto porcentaje de similitudes para el caso de los frutos. El análisis del aceite esencial de hojas de la especie Croton funckianus colectada en Fusagasugá mostró como componentes principales citronelal, 22,8\%; neral, 18,6\%; carvona, 13,4\%, y $\beta$-gurjuneno, 12,2\%; mientras que para la especie bogotana fueron linalool, 25,8\%; $\alpha$-terpineol, 26,9\%; limoneno, 18,9\%, y neral, 18,6\%. Para el caso de los frutos se observaron diferencias en cuanto al porcentaje de los componentes mayoritarios; para la muestra de Fusagasugá: linalool, 25,0\%; borneol, 15,5\%, y neral, 18,6\%, y para la especie de Bogotá: linalool, $22 \%$; borneol, $13 \%$, y neral, 17,2\%.
\end{abstract}

Palabras clave: Croton funckianus, aceites esenciales, cromatografía de gases, índice de Kovats.

\begin{abstract}
A chemical characterization from the essential oil obtained from leaves and fruits of two samples of Croton funckianus that were collected in two different sites (Fusagasugá and Bogotá) was conducted. The analysis was performed using gas chromatography coupled with mass spectrometry (GC-MS), and posteriorly, a comparison between the obtained profiles showed some differences for the leaves and a high percentage of similarity for the fruits. The analysis of the essential oil from the leaves of Croton funckianus collected in Fusagasugá showed as main components citronellal (22.8\%), neral (18.6\%), carvone (13.4\%) and $\beta$-gurjunene (12.2\%); whereas, for $C$. funckianus collected in were linalool (25.8\%), $\alpha$-terpineol (26.9\%), limonene $(18.9 \%)$ and neral $(18.6 \%)$. In the fruit samples, differences in the major components percentages were observed, such that for the Fusagasuga sample were linalool $(25.0 \%)$, borneol $(15.5 \%)$ and neral (18.6\%), and for the Bogotá sample were linalool (22\%), borneol (13\%) and neral (17.2\%).
\end{abstract}

Key words: Croton funckianus, Essential oils, Gas chromatography, Kovats index.

${ }^{a}$ Grupo Integrado de Investigacion en Química y Biología (InQuiBio), Facultad de Ciencias Básicas y Aplicadas, Universidad Militar Nueva Granada.

*Autor de correspondencia: carlos.coy@unimilitar.edu.co 


\section{Introducción}

De acuerdo con la tendencia de las personas de hacer uso de medicamentos de origen natural, para los investigadores en productos naturales se hace indispensable profundizar en el conocimiento de las plantas medicinales, de su composición química y de los factores que puedan hacerla variar, así como conocer la estructura y las propiedades de los principios activos, con miras a poder determinar su posible actividad farmacológica, las indicaciones, contraindicaciones y efectos secundarios, entre otros, para utilizarlas en la prevención y el tratamiento de problemas sanitarios [1].

Los aceites esenciales derivados de plantas han sido muy utilizados desde la antigüedad, porque tienen distintas propiedades etnobotánicas, organolépticas y de bioactividad; es así como parte de las investigaciones actuales se han enfocado en la búsqueda de compuestos que se puedan usar como agentes terapéuticos, con el fin de controlar y tratar diferentes enfermedades derivadas de los microorganismos $[2,3]$. Por otra parte, el uso de los aceites esenciales ha tomado un papel muy importante en esta búsqueda, debido, en gran parte, a que muchas veces la mezcla de componentes terpénicos (en su mayoría oxigenados), obtenidos por su correspondiente caracterización química, usando la técnica de CG-EM, presenta algún tipo de actividad frente a bacterias comunes [4,5], lo cual tiene como objetivo primordial desarrollar alternativas de uso y ampliar las perspectivas para la utilización de los productos naturales, volátiles en este caso en particular, aumentando de esta manera las posibilidades de encontrar nuevos agentes terapéuticos derivados de plantas.

Según Murillo [6] y Cronquist [7], Croton es el segundo género con más riqueza y abundancia dentro de la familia de las Euphorbiaceae; cuenta con un número aproximado de 800 especies, principalmente distribuidas en la región pantropical. En Colombia se registran 83 especies de este género, distribuidas en 19 departamentos; estas especies se han conocido popularmente con los nombres de "sangregado", "zangrado" o "sangre de drago", entre otros, dependiendo de la zona geográfica.

Desde el punto de vista etnobotánico, se han encontrado registros importantes en cuanto al uso, a nivel popular, del exudado de Croton, en las zonas de Centro y Suramérica, debido a sus propiedades antiinflamatorias y antisépticas; de igual manera, también es conocido por ser un regulador homeostático y antidiarreico [8]. Algunas poblaciones indígenas han utilizado el exudado para el tratamiento de fiebres de origen digestivo y para baños vaginales antes del parto, entre otras aplicaciones [9]. Como ejemplo particular, se reporta que el aceite esencial obtenido de las semillas de Croton tiglium ha sido empleado como purgante [10].

Varias especies del género Croton se han convertido en objeto de estudio en los últimos años por parte de diferentes grupos de investigación, posiblemente por la gran riqueza de compuestos que poseen y el potencial como fuente de agentes terapéuticos.

\section{Experimental}

\subsection{Colecta del material}

Se colectaron dos muestras de la especie Croton funckianus; la primera, en una zona de bosque natural ubicado en la vereda Bosachoque (FusagasugáColombia), y la otra, en la localidad de Teusaquillo de Bogotá D.C. (Colombia). Las especies colectadas fueron enviadas al Herbario Nacional Colombiano (COL), del Instituto de Ciencias Naturales (ICN) de la Universidad Nacional de Colombia, sede Bogotá (COL 85051 y 11745).

\subsection{Obtención del aceite esencial de hojas y frutos de $C$. funckianus}

La muestra de hojas frescas fue trasladada al laboratorio de Investigación de la Universidad Militar Nueva Granada, bajo refrigeración, a una temperatura de $4{ }^{\circ} \mathrm{C}$; posteriormente, se extrajo el aceite esencial a $2000 \mathrm{~g}$ de material, tanto de frutos como de hojas, proveniente de cada especie; procedimiento que se realizó en un equipo de destilación por arrastre con vapor durante un tiempo de dos horas. El aceite esencial obtenido ( $2 \mathrm{~mL})$ se separó de la capa acuosa, se secó con sulfato de sodio anhidro y se filtró. Se utilizó una alícuota para determinar su composición química. Luego, se determinaron los índices de Kovats, analizando conjuntamente la muestra con una mezcla de $n$-alcanos $\left(\mathrm{C}_{8}\right.$ a $\left.\mathrm{C}_{22}\right)$. La separación e identificación se realizó en un equipo Hewlett Packard 5973 GC/MS a 70 eV, equipado con un inyector automático, utilizando una columna capilar HP-5MS (30 m, 0,25 mm, 0,25 mm). Un alícuota 
del aceite $(50 \mu \mathrm{L})$ se diluyó en $1 \mathrm{~mL}$ de $n$-hexano para el correspondiente análisis cromatográfico. Se utilizó una temperatura de inyección con una rampa de $50{ }^{\circ} \mathrm{C}-200{ }^{\circ} \mathrm{C}$. La identificación de los componentes químicos fue realizada por comparación de los espectros de masas con las bases de datos NIST 02, Wiley 6 y Adams 2004 [11].

\section{Resultados y discusión de resultados}

En la tabla 1 se muestran los resultados de la composición química del aceite esencial de hojas de la especie Croton funckianus recolectadas en Fusagasugá; en la tabla 2, los resultados de la composición química del aceite esencial de hojas de Croton funckianus recolectadas en Bogotá.

Tabla 1. Componentes volátiles mayoritarios del aceite esencial de hojas de Croton funckianus (Fusagasugá).

\begin{tabular}{clccc}
\hline No. Compuesto & IK Calc.* IK Lit.**[11] & $\begin{array}{c}\text { Área } \\
\text { relativa } \%\end{array}$ \\
\hline 1 & $\alpha$-pineno & 935 & 937 & 1,0 \\
2 & camfeno & 962 & 954 & 0.2 \\
3 & $\beta$-mirceno & 988 & 990 & 0,5 \\
4 & $\alpha$-felandreno & 1001 & 1000 & 0,5 \\
5 & limoneno & 1038 & 1034 & 1,5 \\
6 & linalool & 1100 & 1101 & 2,5 \\
7 & citronellal & 1149 & 1153 & 22,8 \\
8 & borneol & 1178 & 1180 & 0,1 \\
9 & nerol & 1223 & 1227 & 0,1 \\
10 & neral & 1240 & 1246 & 18,6 \\
11 & geraniol & 1248 & 1254 & 2,3 \\
12 & carvona & 1255 & 1257 & 13,4 \\
13 & acetato de nerilo & 1356 & 1357 & 0,2 \\
14 & $\beta$-elemeno & 1390 & 1397 & 0,2 \\
15 & $\beta$-gurjuneno & 1442 & 1441 & 12,2 \\
16 & $\alpha$-humuleno & 1468 & 1469 & 6,3 \\
17 & $\alpha$-guianeno & 1477 & 1475 & 5,6 \\
18 & óxido de cariofileno & 1602 & 1599 & 0,6 \\
19 & biciclogermacreno & 1507 & 1509 & 1,7 \\
20 & $\alpha$-bulneseno & 1532 & 1520 & 0,5 \\
\hline$* I K$ & Calc. Índice de Kovats determinado experimentalmente \\
en columna HP-5. & & & \\
$* *$ IK Lit. Índice Literatura [11]. & & \\
& & &
\end{tabular}

En el análisis por CG-EM de los aceites esenciales fue posible identificar y cuantificar, para la muestra de hojas de Fusagasugá, 20 compuestos, que corresponden al 89,8\%: 63,1\% terpenos, y 26,7\%, sesquiterpenos; los compuestos mayoritarios fueron citronelal, 22,8\%; neral, 18,6\%; carvona, $13,4 \%$, y $\beta$-gurjuneno, $12,2 \%$. Mientras que en la muestra de Bogotá se identificaron 11 componentes, que correspondieron al $97 \%$, todos compuestos terpénicos, y se encontró que los compuestos mayoritarios
Tabla 2. Componentes volátiles mayoritarios del aceite esencial de hojas de C. funckianus (Bogotá).

\begin{tabular}{clccc}
\hline No. Compuesto & IK Calc.* & IK Lit.** [11] & $\begin{array}{c}\text { Área } \\
\text { relativa\% }\end{array}$ \\
\hline 1 & 2-heptanol & 908 & 908 & 0,8 \\
2 & $\alpha$-pineno & 935 & 937 & 1,0 \\
3 & sabineno & 979 & 973 & 1,3 \\
4 & 1,8 -cineol & 1038 & 1033 & 2,2 \\
5 & limoneno & 1038 & 1034 & 18,9 \\
6 & linalool & 1100 & 1101 & 25,8 \\
7 & a-terpineol & 1192 & 1195 & 26,9 \\
8 & borneol & 1178 & 1180 & 0,9 \\
9 & neral & 1240 & 1246 & 18,6 \\
10 & bicicloelemeno & 1271 & 1272 & 0,4 \\
11 & acetato de nerilo & 1356 & 1357 & 0,2 \\
\hline
\end{tabular}

*IK Calc. Índice de Kovats determinado experimentalmente en columna HP-5.

**IK Lit. Índice Literatura [11].

fueron linalool, 25,8\%; $\alpha$-terpineol, 26,9\%; limoneno, $18,9 \%$, y neral, $18,6 \%$.

Los datos anteriores demuestran que los aceites esenciales de hojas de Croton son producidos por todas las partes del planta, en especial hojas y frutos, y por lo general representan el 1,2\% [12] del peso del material vegetal seco; este aceite está constituido por terpenos y sesquiterpenos, principalmente. Para la especie en estudio es la primera vez que se reporta la caracterización del aceite de hojas y la comparación de sus componentes de acuerdo con el sitio de recolección.

Vale la pena mencionar que se ha encontrado que el aceite de Croton huberii tiene $\alpha$-pineno, $\beta$ cariofileno, germacreno D y óxido de cariofileno [13]; que los aceites de Croton jacobibensis, Croton rhamnifolius y Croton musicapa tienen alcanfor, guiaiol y bulneseno, y que Croton cajucara tiene como componente mayoritario el linalool, utilizado como agente antimicrobiano [14]. Otras especies que tienen el compuesto linalool como mayoritario son: Croton sacaquinha, 5,7\%; Croton lanjouwensis, 26,7\%; Croton stellulifer y Croton zambesicus [15]. Resulta interesante que la especie bogotana tenga este compuesto como mayoritario, en un porcentaje de $25,8 \%$.

Por su parte, el componente mayoritario de la especie de Fusagasugá fue el citronellal $(22,8 \%)$, un monoterpeno oxigenado muy utilizado en la industria cosmética, farmacéutica y de alimentos, que tiene propiedades antiinflamatorias [16], antioxidantes, 
antidepresivas [17], antinociceptivas [18] y antifúngicas, entre otras [19].

En la tabla 3 se muestran los resultados de la composición química del aceite esencial de frutos de la especie $C$. funckianus recolectada en Fusagasugá; en la tabla 4 se presentan los resultados de la composición química del aceite esencial de frutos de Croton funckianus recolectado en Bogotá.

Tabla 3. Componentes volátiles mayoritarios del aceite esencial de frutos de Croton funckianus (Fusagasugá).

\begin{tabular}{clccc}
\hline No. & Compuesto & IK Calc.* & IK Lit.** [11] & $\begin{array}{c}\text { Área } \\
\text { relativa } \%\end{array}$ \\
\hline 1 & $\alpha$-pineno & 935 & 937 & 1,0 \\
2 & canfeno & 962 & 954 & 0,2 \\
3 & $\beta$-mirceno & 988 & 990 & 0,5 \\
4 & $\alpha$-felandreno & 1001 & 1000 & 0,5 \\
5 & limoneno & 1038 & 1034 & 15,7 \\
6 & linalool & 1100 & 1101 & 25,0 \\
7 & citronellal & 1149 & 1153 & 12,5 \\
8 & borneol & 1178 & 1180 & 15,5 \\
9 & nerol & 1223 & 1227 & 0,1 \\
10 & neral & 1240 & 1246 & 18,6 \\
11 & geraniol & 1248 & 1254 & 2,3 \\
12 & carvona & 1255 & 1257 & 0,2 \\
13 & acetato de nerilo & 1356 & 1357 & 0,2 \\
14 & $\beta$-elemeno & 1390 & 1397 & 0,2 \\
15 & $\beta$-gurjuneno & 1442 & 1441 & 0,2 \\
16 & $\alpha$-humuleno & 1468 & 1469 & 0,2 \\
17 & $\alpha$-guianeno & 1477 & 1475 & 0,2 \\
\hline
\end{tabular}

*IK Calc. Índice de Kovats determinado experimentalmente en columna HP-5.

** IK Lit. Índice Literatura [11]

El análisis correspondiente al aceite esencial de frutos mostró una similitud bastante interesante, ya que el perfil de cada uno de los aceites resultó ser muy parecido, variando para cada caso el porcentaje de componentes mayoritarios; para el aceite esencial de la especie de Fusagasugá se encontró que tiene 17 componentes (77,6\%): 76,6\% monoterpenos y $1,0 \%$ sesquiterpenos; los componentes mayoritarios fueron linalool, 25,0\%; borneol, $15,5 \%$, y neral, 18,6\%. La especie de Bogotá tiene 17 componentes (89,6\%): $87,7 \%$ monoterpenos y $2,0 \%$ sesquiterpenos; los principales componentes fueron los mismos que para el aceite anterior, lo que cambió fue el porcentaje encontrado para cada uno: linalool, $22 \%$; borneol, $13 \%$, y neral, 17,2\%.

El componente neral fue encontrado en los cuatro aceites, con un porcentaje de composición muy similar, entre $17,2 \%$ y $18,6 \%$; este compuesto se caracteriza por sus propiedades antifúngicas y
Tabla 4. Componentes volátiles mayoritarios del aceite esencial de frutos de Croton funckianus (Bogotá).

\begin{tabular}{clccc}
\hline No. & Compuesto & IK Calc.* & IK Lit.** [11] & $\begin{array}{c}\text { Área } \\
\text { relativa \% }\end{array}$ \\
\hline 1 & $\alpha$-pineno & 935 & 937 & 1,0 \\
2 & canfeno & 962 & 954 & 0,2 \\
3 & $\beta$-mirceno & 988 & 990 & 0,5 \\
4 & $\alpha$-felandreno & 1001 & 1000 & 0,5 \\
5 & limoneno & 1038 & 1034 & 15,7 \\
6 & linalool & 1100 & 1101 & 22,0 \\
7 & citronellal & 1149 & 1153 & 12,5 \\
8 & borneol & 1178 & 1180 & 13,0 \\
9 & nerol & 1223 & 1227 & 0,1 \\
10 & neral & 1240 & 1246 & 17,2 \\
11 & geraniol & 1248 & 1254 & 2,3 \\
12 & carvona & 1255 & 1257 & 1,0 \\
13 & acetato de nerilo & 1356 & 1357 & 1,0 \\
14 & $\beta$-elemeno & 1390 & 1397 & 1,0 \\
15 & $\beta$-gurjuneno & 1442 & 1441 & 0,2 \\
16 & $\alpha$-humuleno & 1468 & 1469 & 0,2 \\
17 & $\alpha$-guianeno & 1477 & 1475 & 0,2 \\
\hline *IK Calc. Índice de Kovats determinado experimentalmente \\
en columna HP-5. \\
**IK Lit. Índice Literatura [11].
\end{tabular}

antimicrobianas [20]. Dentro de los estudios anteriores sobre especies del género Croton no existen reportes de este compuesto como componente mayoritario, lo que posiblemente podría tenerse en cuenta para estudios quimiotaxonómicos posteriores.

Luego de observar los datos obtenidos para la caracterización de cada uno de los aceites derivados de las hojas, se nota que aunque son la misma especie, existen algunas diferencias en cuanto a los componentes volátiles de cada uno; en primera instancia, y de acuerdo con el cromatograma obtenido, el aceite de hojas que proviene de Fusagasugá muestra mayor cantidad de componentes, teniendo en cuenta que se realizaron bajo las mismas condiciones, respecto al cromatograma del aceite de la especie bogotana; en cambio, la similitud que tienen los aceites derivados de los frutos es bastante alta, y en lo único que varían es en el porcentaje de composición.

\section{Conclusiones}

El análisis por CG-EM de los aceites esenciales de hojas y de frutos de las dos muestras de Croton funckianus, recolectadas en sitios diferentes, una en Fusagasugá y la otra en Bogotá, permitió determinar que los componentes de las hojas varían de acuerdo con el sitio de colecta, destacándose la producción de componentes del tipo monoterpenoide y 
sesquiterpenoide; la comparación también señala que las hojas de la muestra de Fusagasugá tienen más componentes que la de Bogotá, lo que hace pensar que, aunque son la misma especie, el entorno y demás variables ecosistémicas, probablemente, repercuten en la producción de los componentes volátiles, lo cual se ve reflejado claramente en los resultados del estudio. Al contrario, los componentes de los frutos son muy similares, y varían solo respecto al porcentaje de composición, en lo demás se encontró un mismo perfil cromatográfico. Resultaría interesante realizar en estudios posteriores la comparación de especies de Croton funckianus recolectadas en otros lugares de la región cundiboyacense, con el fin de observar la posible variabilidad química de sus aceites esenciales, y así enriquecer los estudios fitoquímicos, con el fin de establecer los usos potenciales de esta especie ampliamente distribuida en la región.

\section{Agradecimientos}

Al grupo integrado de Investigaciones en Química y Biología, de la Facultad de Ciencias Básicas y Aplicadas; a la Vicerrectoría de Investigaciones de la Universidad Militar Nueva Granada, por la financiación del proyecto CIAS 1473, mediante convocatoria interna.

\section{Referencias}

[1] J. Bruneton, Farmacognosia Fitoquímica plantas medicinales. $2^{a}$ edición, Zaragoza, España: Acribia, 2001.

[2] S. Gibbons, "Anti-staphylococcal plant natural products", Natural Products Research, vol. 21, pp. 263-277, 2004.

[3] S. Gibbons, "Plants as a source of bacterial resistance modulators and anti-infective agents", Phytochemistry Reviews, vol. 4, pp. 63-78, 2005.

[4] S. S. Eteghad, H. Mirzaei, S.F. Pour, S. Kahnaui, "Inhibitory Effects of endemic, Thymus vulgaris and Menta piperita Essential Oils on Escherichia coli O157:H7”, Research Journal of Biology Science, vol. 4, no. 3, pp. 340-344, 2009.

[5] A. H. Jarrar, K. Adwan, "Antibacterial activity of Rosmarinus officinalis L. alone and in combination with cefuroxime against methicillin-resistant Staphylococcus aureus", Asian Pacific Journal of Tropical Medicine, vol. 1, pp. 121-123, 2010.

[6] J. Murillo, "Composición y distribución del género Croton (Euphorbiaceae) en Colombia, con cuatro especies nuevas", Revista Caldasia, vol. 21, n. ${ }^{\circ}$ 2, pp. 141-166, 1993.

[7] A. Cronquist, Integrated System of classification of Flowering plants. New York Botanical Garden. Bronx. NY. 1981.

[8] L. Pieters, D. Bruyne, M. Claeys, J. Vlietinch, "Isolation of the dihydrobenzofuran lignan from the South American dragon's blood (Croton sp.) as an ininhibitor of cell proliferation", Journal of Natural Products, vol. 56, no. 6, pp. 899906, 1993.

[9] J. H. Tamariz, R. Capcha, E. Palomino, J. Aguilar, "Actividad antibacteriana de la Sangre de Grado (Croton lechleri) frente al Helicobacter pylori”, Revista Médica Herediana, vol. 14, no. 2, pp. 81-88, 2003.

[10] J. C. Tsai, S. L. Tsai, W. C. Chang, "Effect of Ethanol Extracts of Three Chinese Medicinal Plants with Laxative Properties on Ion Transport of the Rat Intestinal Epithelia”, Biological Pharmaceutical Bulletin, vol. 27, pp. 162-165, 2004.

[11] P. Adams, Identification of essential oil components by Gas Chromatography/Mass Spectrometry, $4^{\text {th }}$ Ed., Allured Publishing, Carol Stream, IL, 2007.

[12] E. Angélico, J. Costa, O. Rodriguez, E. De Lima, R.S, "Medeiros. composição química do óleo essencial das folhas de Croton blanchetianus (Baill): Resultados Preliminares", Revista de Biologia e Farmácia, vol. 5, no. 2, pp. 44-49. 2011.

[13] J. Rojas, A. Buitrago, L. Rojas, J. Cárdenas, J. Carmona, "Chemical composition of the essential oil of Croton huberi Steyerb. Euphorbiaceae Collected from Táchira Venezuela", Journal of Essential Oil Bearing Plants, vol. 16, no. 5, pp. 646-650, 2013.

[14] I. A. Neves, C.A. da Camara, "Acaricidal activity against Tetranychus urticae and essential oil composition of four Croton species from 
Caatinga biome in northeastern Brazil", Natural Products Communication, vol. 6, no. 6, pp. 893-899, 2011.

[15] N. Radulovik, E. Mananjarasoa, L. Harinantenaina, A. Yoshinori, "Essential oil composition of four Croton species from Madagascar and their chemotaxonomy", Biochemical Systematics and Ecology, vol. 34, pp. 648-653, 2006.

[16] M.S. Melo, A.G. Guimaraes, M.F. Santana, R.S. Siqueira, A.C de Lima, A.S. Dias, M.R. Santos, A.S. Onofre, J. S. Quintans, D. P de souza, J.R. Almeida, C.S. Estevam, B.S. Araujo, J. Quintans, "Antiinflamatory and redox protective activities of citronellal", Biological Research, vol. 44, no. 4, pp. 363-368, 2012.

[17] F.N. Victoria, R. Anversa, F. Penteado, M. Castro, E.J. Lenardao, L. Savegnago, "Antioxidant antidepressant-like activities of semisynthetic alpha phelineseno-citronellal", European
Journal of Pharmacology, vol. 742, pp. 131138, 2014.

[18] L. Quintans, R. Fagundes, F. Freitas, J. Fonseca, F.A da Silva, A. De Souza, J.P. Almeida, M.S. Melo, D.P. de Souza, L.R. Bonjardim, D.P. Gelain, "Antinociceptive Action and Redox Properties of Citronellal, and essential oil present in lemongrass", Journal of Medicinal Food, vol. 14, no. 6, pp. 630-639, 2011.

[19] R. Wagner, M. Ootani, S. Donizeti, T. Ferreira, M. dos Santos, G. dos Santos, "Fumigant Antifungal activity of Corymbia citriodora and Cymbopogon nardus Essential oils and citronellal against Three Fungal Species", The Scientific World Journal, pp. 1-8, 2014.

[20] C. Da Silva, S. Guterres, V. Weisheimer, E. Schapoval, "Antifungal activity of the lemongrass oil and citral against candida spp", Brazilian Journal of Infectious Diseases, vol. 12, no. 1, pp. 63-66, 2008. 